\title{
Thermoresponsive dynamic BAB block copolymer networks synthesized by aqueous PISA in one-pot ${ }^{\dagger}$
}

Pauline Biais ${ }^{\mathrm{a}}$, Marie Engel ${ }^{\mathrm{a}}$, Olivier Colombani ${ }^{\mathrm{b}}$, Taco Nicolai ${ }^{\mathrm{b}}$, François Stoffelbach ${ }^{\mathrm{a}}$, Jutta Rieger $^{\mathrm{a}, *}$

a Sorbonne Université, CNRS, Institut Parisien de Chimie Moléculaire, UMR 8232, Polymer

Chemistry Team, 4 Place Jussieu, 75252 Paris Cedex 05, France

E-mail: jutta.rieger@sorbonne-universite.fr

b Institut des Molécules et Matériaux du Mans (IMMM), UMR 6283 CNRS Le Mans

Université, Avenue Olivier Messiaen, 72085 Le Mans Cedex 9, France

FIGURE FOR TOC

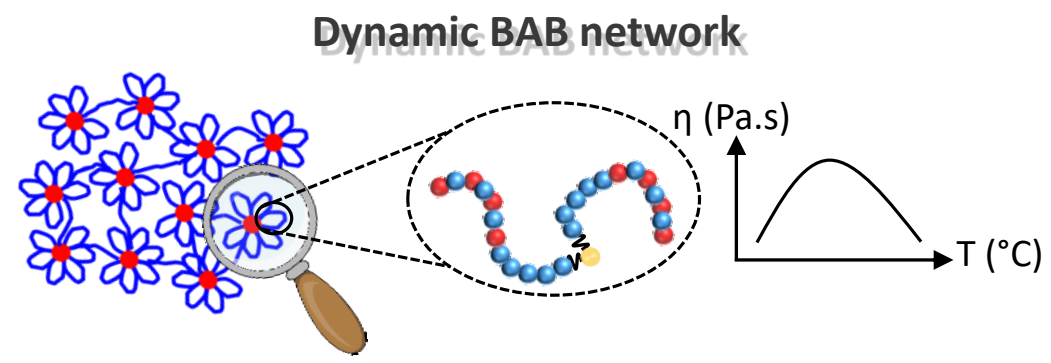

\section{Text for TOC}

The incorporation of neutral hydrophilic monomer units in the hydrophobic $\mathrm{B}$ blocks of BAB copolymers produces transient networks exhibiting a thermoresponsive behavior with a maximum of viscosity in water.

\footnotetext{
$\dagger$ This manuscript is in honor of the 50-year anniversary of the French Polymer Group (Groupe Français des Polymères - GFP)
} 


\begin{abstract}
We report a straightforward process to produce well-defined dynamic networks of associative $\mathrm{BAB}$ triblock copolymers through two successive polymerization steps conducted in one-pot and exclusively in water. The copolymers are composed of a hydrophilic $\operatorname{poly}(N, N-$ dimethylacrylamide) A block extended in a typical RAFT-PISA dispersion formulation with two lateral associative statistical poly(diacetone acrylamide-co- $N, N$-dimethylacrylamide) B blocks. The viscosity of the samples is strongly modified by the $N, N$-dimethylacrylamide (DMAc) content in the B block. This is attributed to the formation of a transient network for which the exchange dynamics of the B blocks between micelles and/or the number of bridges within the network depend on the DMAc content. Rheology analyses showed that transient networks are formed that exhibit a thermoresponsive behavior with a maximum viscosity as a function of temperature, presumably caused by a maximum of bond lifetime and/or number of elastically active chains.
\end{abstract}

\title{
Introduction
}

In the last decade, polymeric systems that exhibit temperature-induced changes in viscosity or true sol-gel transitions, such as thermoresponsive physical hydrogels, have found large interest for various applications. ${ }^{1,2}$ In particular in the biomedical field, ${ }^{3}$ thermoresponsive hydrogels have been designed as smart drug delivery systems that are able to release the drug on demand by a reversible volume-phase transition or sol-gel transition triggered by a change in temperature..$^{4-7}$ Another important application of thermoresponsive hydrogels is their use as injectable gels, for instance for tissue engineering: liquid at room temperature they become gellike at body temperature. ${ }^{8-10}$

Thermoresponsive hydrogels are generally based on water-soluble polymeric chains to which thermoresponsive moieties are linked, either grafted along the polymer chain or at their end. ${ }^{1,2}$ One of the simplest architectures are BAB triblock copolymers, where the A block is watersoluble and the B block is a thermoresponsive polymer, exhibiting either a lower or upper critical solution temperature (LCST or UCST). ${ }^{11-13}$ The LCST behaviour results from a coilglobule transition of the polymer chains that occurs above a critical and concentrationdependant temperature caused by a decrease in solvation of the polymer. The first and most studied LCST-type polymer is poly( $N$-isopropyl acrylamide) (PNIPAm), which exhibits a sharp coil-globule transition and a LCST at $32{ }^{\circ} \mathrm{C} .{ }^{14} \mathrm{BAB}$ triblock copolymers with PNIPAm B 
blocks, for instance PNIPAm- $b$-PDMAc- $b$-PNIPAm triblock copolymers (with PDMAc = $\operatorname{poly}(N, N$-dimethylacrylamide $)$ have been used to form thermoresponsive hydrogels, for which the gelation temperature and the mechanical properties depend on the polymer concentration and the length of the middle block. ${ }^{9}$

The behavior in aqueous medium of B AB triblock copolymers with permanently hydrophobic B blocks is well known as long as thermodynamic equilibrium is reached. ${ }^{15,16}$ In dilute solution, BAB-type copolymers aggregate into flower-like micelles with the A block forming loops around hydrophobic cores formed by the B blocks. Upon increase of the concentration, some loops become bridges and individual micelles interconnect into larger and larger aggregates, which eventually percolate into a macroscopic water-swollen network (Scheme 1).

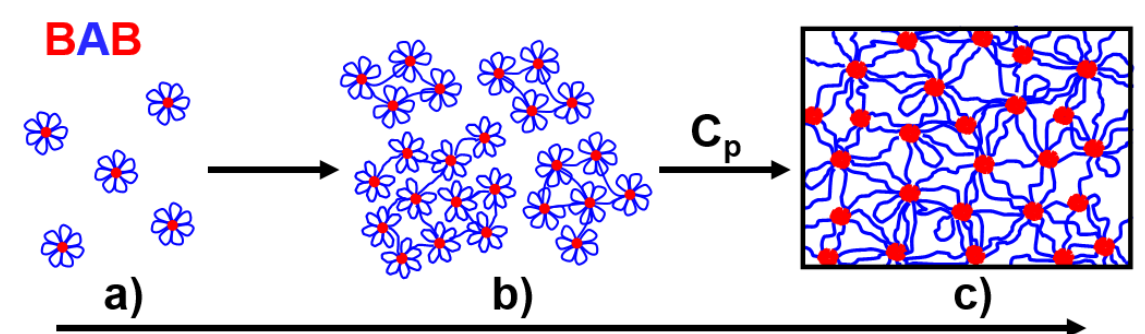

Increasing polymer concentration

Scheme 1. Concentration dependent self-assembly of BAB triblock copolymers: a) individual flower-like micelles, b) finite size aggregates of connected flower-like micelles and c) 3Dspanning transient network of connected flower-like micelles above the percolation concentration. (A block in blue, B blocks in red)

For so-called "dynamic systems" at equilibrium, the exchange of the B blocks between the hydrophobic micellar cores occurs continuously and the rheological properties of the BAB dispersion are controlled both by the exchange rate of the B blocks and the amount of bridges between micelles. Indeed, the relaxation time $\tau$ of the network is directly correlated to the exchange time of the B blocks, whereas the modulus $\left(\mathrm{G}_{\mathrm{e}}\right)$ is proportional to the amount of elastically active chains (i.e. chains forming bridges according to the theory of the rubber elasticity). ${ }^{15-20}$ The viscosity of the network $\eta$ is the product of $\tau$ and $G_{e}$ and is therefore impacted both by the exchange rate of the B blocks and by the number of bridges.

It must be stressed that most amphiphilic block copolymers form out-of-equilibrium, so-called "frozen", structures in aqueous medium: the exchange of B blocks is too slow compared to the 
experimental time-window because the B blocks are not sufficiently mobile (high $\mathrm{T}_{\mathrm{g}}$ ) and/or too hydrophobic to exchange. ${ }^{21}$ It has however been shown that the exchange dynamics of the B blocks can be controlled by the insertion of hydrophilic units within the associative B blocks, leading to polymers exhibiting $\mathrm{pH},{ }^{22-25}$ or more rarely temperature-sensitive ${ }^{25}$ rheological properties.

In terms of synthesis methods, amphiphilic block copolymers are traditionally obtained through living/controlled polymerization by successive polymerization steps performed in non-selective organic solvents, where the polymer does not self-assemble. After a purification step (generally by precipitation), the polymer is isolated as a powder. Its self-assembly in water generally requires complex additional (preparation) steps using organic cosolvents. ${ }^{21,26}$ With the development of polymerization-induced self-assembly (PISA), mainly by Reversible Addition Fragmentation chain Transfer (RAFT), ${ }^{27}$ the synthesis of amphiphilic block copolymers in water has become possible. In a first synthesis step, a water-soluble macroRAFT agent A is synthesized in solution. In a second polymerization step, the actual PISA step, a second block $\mathrm{B}$ is added in heterogeneous polymerization conditions, as this block is insoluble in water. ${ }^{28-34}$ In contrast to conventional syntheses, such PISA syntheses are greener and safer, and avoid time-consuming isolation/dissolution steps because the polymer is directly formed in aqueous medium where it self-assembles simultaneously. While most of the PISA literature concerns the synthesis of amphiphilic diblock copolymers, a few examples of BAB triblock copolymers have also been reported. ${ }^{35-37}$ In 2018 , we reported ${ }^{38}$ the first successful synthesis of welldefined BAB triblock copolymers by PISA in water leading to colloidally stable dispersions. In that work, a poly $(N, N$-dimethylacrylamide) (PDMAc) macroRAFT agent was first synthesized in DMF and then chain-extended with two poly(diacetone acrylamide) (PDAAm) lateral blocks by RAFT polymerization in water. In a subsequent study, we demonstrated that nanometric aggregates of bridged flower-like micelles were formed in stirred polymerization conditions. ${ }^{39}$ The latter resulted from the formation of a frozen network of bridged micelles, which was irreversibly broken into tiny pieces through the mechanical stirring.

In this work, we aimed at synthesizing dynamic networks of BAB triblock copolymers based on the same monomer units, DMAc and DAAm, by PISA. Our strategy to render the formerly kinetically frozen networks dynamic relies on the insertion of hydrophilic DMAc monomer units in the PDAAm segments. Tuning the amount of comonomer in the segment should allow controlling the exchange dynamics and thereby the rheological properties of the complex fluids. The incorporation of hydrophilic monomer units into the solvophobic blocks of BAB triblock 
copolymers has already been used as a way to control the rheological properties of the polymers as mentioned above. However, ionizable hydrophilic units were used in all reports. No attempt has been reported to tune the rheological properties of such polymers using neutral hydrophilic comonomer units. It has been postulated that the incorporation of neutral solvophilic monomer units in the B blocks of amphiphilic AB diblock copolymers would increase the chain mobility in $\mathrm{AB}$ diblock copolymer assemblies, favoring morphological transitions. ${ }^{40-42}$ However, no corresponding $\mathrm{BAB}$ triblocks were synthesized and their rheological properties were consequently not studied. In order to synthesize such dynamic BAB networks in water, we developed a straightforward one-pot strategy, which uses water as the only solvent, and which consists of two consecutive polymerization steps. The first step consists in the synthesis of the bifunctional PDMAc A block in water. The A block is then chain extended in the second step through a typical PISA aqueous dispersion copolymerization of a mixture of DAAm and DMAc (in various proportions). We not only describe for the first time an entirely water-borne synthesis of such dynamic $\mathrm{BAB}$ assemblies, but we also show that the aqueous dispersion possesses an unexpected thermo-responsiveness presenting a maximum viscosity.

\section{Experimental part}

\section{Materials}

$N, N$-Dimethylacrylamide (DMAc, $>99 \%$, Aldrich) was distilled under reduced pressure before use. 1,3,5-Trioxane (99\%, Aldrich), 4,4'-azobis(4-cyanopentanoic acid) (ACPA, $\geq 98 \%$ Aldrich), sodium hydrogen carbonate $\left(\mathrm{NaHCO}_{3},>99.7 \%\right.$ Aldrich), diacetone acrylamide (DAAm, 99\%, Aldrich), were used as received. 3,5-Bis[2-(n-butyltrithiocarbonato)propionyloxy]benzoic acid $\left(\mathrm{C}_{4}-\mathrm{TTC}\right)_{2}$-BA was synthesized as reported before with a purity of $97 \% .{ }^{43}$ Deionized water ( $\rho>18 \mathrm{M} \Omega . \mathrm{cm}$ ) was used for all dispersion polymerizations.

\section{All-water two-step synthesis of [C4-P(DAAm-co-DMAc)-b-PDMAc $]_{2}-\mathrm{BA}$ copolymer networks}

\section{$I^{\text {st }}$ polymerization step: Synthesis of poly(N,N-dimethylacrylamide) macroRAFT agents}

Polymerizations of DMAc in water were initiated by ACPA at $70{ }^{\circ} \mathrm{C}$, in the presence of the RAFT agent ( $\left.\mathrm{C}_{4}-\mathrm{TTC}\right)_{2}$-BA (see Scheme 1). In a typical experiment (Table S1, entry II bis), $85.2 \mathrm{mg}$ of $\left(\mathrm{C}_{4} \text {-TTC }\right)_{2}$-BA $(0.14 \mathrm{mmol})$ was placed in a $25 \mathrm{~mL}$ septum-sealed flask. A small 
amount (123 mg, $1.36 \mathrm{mmol})$ of 1,3,5-trioxane was added as an internal reference for the determination of the monomer consumption by ${ }^{1} \mathrm{H}$ NMR (by the relative integration of the protons of the 1,3,5-trioxane at $5.1 \mathrm{ppm}$ and the vinylic protons of DMAc at 6.7, 6.2 and $5.6 \mathrm{ppm}$ in acetone- $\left.\mathrm{d}_{6}\right)$. A solution of ACPA $\left(11.4 \mathrm{mg}, 4.07 \times 10^{-2} \mathrm{mmol}\right)$ in $0.75 \mathrm{~g}$ of DMAc was prepared. $0.27 \mathrm{~g}$ of this solution $\left(1.46 \times 10^{-2} \mathrm{mmol}\right.$ of ACPA and $2.67 \mathrm{mmol}$ of DMAc) was added in the flask containing the RAFT agent. Then, $2.53 \mathrm{~g}$ of DMAc (25.5 mmol) was added and the solution was purged with argon for $25 \mathrm{~min}$ in an ice bath. The polymerization was then started in bulk by heating the reaction mixture at $70{ }^{\circ} \mathrm{C}$ in a preheated oil bath under stirring. After $37 \mathrm{~min}$, when the solution became too viscous for a homogeneous stirring (corresponding to a monomer conversion around 50\%), $11 \mathrm{~mL}$ of degassed water were added with a syringe, leading to a monomer percentage of $20 \mathrm{wt} \%$. The kinetics of the polymerization were followed by ${ }^{1} \mathrm{H}$ NMR. After $105 \mathrm{~min}, 90 \%$ monomer conversion was reached and the polymerization was stopped by immersion of the flask in iced water and exposure to air. The polymer was characterized by ${ }^{1} \mathrm{H}$ NMR in acetone- $\mathrm{d}_{6}$ and size exclusion chromatography (SEC) in $\mathrm{DMF}\left(+\mathrm{LiBr}, 1 \mathrm{~g} \mathrm{~L}^{-1}\right)$. The polymer solution was then used in the $2^{\text {nd }}$ polymerization step without further purification.

\section{$2^{\text {nd }}$ polymerization step: Aqueous dispersion copolymerization of DMAc and DAAm (PISA)}

All the aqueous dispersion copolymerizations of DAAm and DMAc were performed at $70{ }^{\circ} \mathrm{C}$ at a stirring speed of $500 \mathrm{rpm}$. The amount of monomer (DAAm and DMAc) was adjusted to reach the targeted molar fraction of DMAc in the hydrophobic block, keeping constant the total monomer concentration (at $12 \mathrm{wt} \%$ relative to the total mass) and setting the initiator concentration at $0.76 \mathrm{mmol} \mathrm{L}^{-1}$. The desired ionization degree of the benzoic acid group $(\alpha)$ was reached by adjusting the $\mathrm{pH}$ with an aqueous solution of $\mathrm{Na}_{2} \mathrm{CO}_{3}(3 \mathrm{mM})$.

In a typical experiment (Table 1, entry 180-100-33), $540 \mathrm{mg}$ of DAAm (3.19 mmol) and 48 mg of 1,3,5-trioxane were dissolved in $49 \mu \mathrm{L}$ of a stock solution of ACPA in water (concentration of $20 \mathrm{~g} \mathrm{~L}^{-1}$ neutralized by 3 molar equivalent of $\mathrm{NaHCO}_{3}$ ) and $0.87 \mathrm{~mL}$ of deionized water. $4.31 \mathrm{~g}$ of the aqueous solution of the PDMAc macroRAFT agent II bis obtained in the $1^{\text {st }}$ polymerization step $\left(M_{\mathrm{n}, \mathrm{NMR}}=18.4 \mathrm{~kg} \mathrm{~mol}^{-1}, 4.35 \times 10^{-2} \mathrm{mmol}\right)$ were added, which contained $86.1 \mathrm{mg}$ of residual DMAc $(0.87 \mathrm{mmol})$. Then, $69.5 \mathrm{mg}$ of DMAc $(0.7 \mathrm{mmol})$ were added and the $\mathrm{pH}$ was adjusted to 5 with an aqueous solution of $\mathrm{Na}_{2} \mathrm{CO}_{3}(3 \mathrm{mM})$. The mixture was degassed for $40 \mathrm{~min}$ with argon in an ice bath, and then heated to $70{ }^{\circ} \mathrm{C}$ in an oil bath, under stirring. The polymerization was stopped by immersion in iced water and exposure to air. The monomer conversions were determined by ${ }^{1} \mathrm{H}$ NMR in acetone- $\mathrm{d}_{6}$ by the relative 
integration of the protons of the 1,3,5-trioxane at $5.1 \mathrm{ppm}$ and the vinylic protons of DAAm at $5.5 \mathrm{ppm}$ and of DMAc at 6.7 and $5.6 \mathrm{ppm}$. The [C 4 -P(DAAm-co-DMAc)- $b-\mathrm{PDMAc}]_{2}-\mathrm{BA}$ dispersion was characterized without further purification.

\section{Characterizations techniques}

$p H$. The $\mathrm{pH}$ value of the aqueous dispersions was probed by a $\mathrm{pH}-$ meter (Mettler Toledo DL50 Graphix) using a micro-pH electrode (Mettler Toledo DGi101-SC).

$p K_{a}$ and ionization degree ( $\alpha$ ). The apparent $\mathrm{pK}_{\mathrm{a}}$ value (i.e. $\mathrm{pK}_{\mathrm{a}}$ for $\alpha=50 \%$ ) of a PDMAc macroRAFT agent $\left(M_{\mathrm{n}, \mathrm{NMR}}=8.8 \mathrm{~kg} \mathrm{~mol}^{-1}\right)$ was determined on a $1 \mathrm{mM}$ solution at $20{ }^{\circ} \mathrm{C}$ by titration of the benzoic acid units with a $0.01 \mathrm{M} \mathrm{NaOH}$ aqueous solution (see Figure S1) using a pH-M20 Lab pH-meter equipped with a pH combination electrode :

\section{(Equation 1)}

$$
\alpha=\frac{K a}{K a+10^{-p H}}
$$

with $\quad \alpha$ : ionization degree

Ka: apparent acid dissociation constant

${ }^{1} H$ NMR. DMAc and DAAm conversions were followed by ${ }^{1} \mathrm{H}$ NMR in acetone- $\mathrm{d}_{6}$ at room temperature with a Bruker 300 or $400 \mathrm{MHz}$ spectrometer in 5-mm diameter tubes.

Variable Temperature ${ }^{1} \mathrm{H}$ NMR. ${ }^{1} \mathrm{H}$ NMR spectra were recorded using a $600 \mathrm{MHz}$ Bruker. The aqueous dispersion was dried under reduced pressure and the resulting solid dispersed in $\mathrm{D}_{2} \mathrm{O}$ at $2.5 \mathrm{wt} \%$ in 5-mm diameter tubes. Spectra were recorded increasing the temperature from 5 ${ }^{\circ} \mathrm{C}$ to $80{ }^{\circ} \mathrm{C}$ with an equilibration time of $10 \mathrm{~min}$ at each temperature.

SEC. Size exclusion chromatography (SEC) analyses were carried out on two PSS GRAM 1000 A columns $\left(8 \times 300 \mathrm{~mm}\right.$; separation limits: 1 to $\left.1000 \mathrm{~kg} \mathrm{~mol}^{-1}\right)$ and one PSS GRAM $30 \AA$ ( $8 \times$ $300 \mathrm{~mm}$; separation limits: 0.1 to $10 \mathrm{~kg} \mathrm{~mol}^{-1}$ ) coupled with a differential refractive index (RI) detector and a $\mathrm{UV}$ detector. $\mathrm{DMF}\left(+\mathrm{LiBr}, 1 \mathrm{~g} \mathrm{~L}^{-1}\right)$ at $60^{\circ} \mathrm{C}$ was used as the mobile phase with

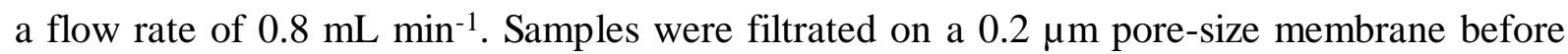
injection. The dispersity $\left(Ð=M_{\mathrm{w}} / M_{\mathrm{n}}\right)$, the number-average molar mass $\left(M_{\mathrm{n}}\right)$ and the weightaverage molar mass $\left(M_{\mathrm{w}}\right)$ were calculated from the RI signals by a calibration curve based on PMMA standards with OmniSEC 5.11 software. 
TEM. Transmission electron microscopy (TEM) was used to determine the morphology and size of the polymer nanoparticles and performed either on a JEOL JEM 2010 UHR microscope or on a JEOL JEM 2100Plus operating at $200 \mathrm{keV}$. The images were collected with a $4008 \times$ 2672 pixel CCD camera (Gatan Orius SC1000). The aqueous dispersions were diluted in water to $0.1 \mathrm{wt} \%$ prior to analysis and then deposited at room temperature on a carbon-coated copper grid. For stained TEM, phosphotungstic acid (0.05 wt\%) was used as staining agent.

Cryo-TEM. Cryogenic transmission electron microscopy (cryo-TEM) was used to determine the morphology and size of the polymer nanoparticles. The samples were diluted in water to 1 wt\% prior to analysis. According to protocols reported elsewhere, ${ }^{44,45}$ thin liquid films of particle dispersions were prepared at room temperature by depositing $4 \mu \mathrm{L}$ of the diluted sample and successive blotting. The thin liquid films were flash frozen in liquid ethane and observed at $-180{ }^{\circ} \mathrm{C}$ on a JEOL JEM-2100 $\mathrm{LaB}_{6}$ microscope operating at $200 \mathrm{kV}$ under low-dose conditions (10 electrons $\AA^{-2} \mathrm{~s}^{-1}$ ). Digital images were recorded on an Ultrascan $1000,2 \mathrm{k}$ x $2 \mathrm{k}$ CCD camera (Gatan, USA).

Vial inversion tests. The macroscopic aspect and the viscosity of the dispersions were observed by the naked eye at 5,25 and $70{ }^{\circ} \mathrm{C}$. After being set at the desired temperature for 5 minutes, the vials containing the dispersions were inverted as shown in the video available in the Supporting Information.

Rheology. Measurements were performed using two controlled-stress rheometers (ARG2 and AR2000, TA Instruments, Crawley, UK) equipped with a cone-plate geometry $\left(40 \mathrm{~mm}, 2^{\circ}\right)$. Silicon oil was used to cover the free sample surface in order to avoid water evaporation during the measurements. The temperature was controlled within $0.1{ }^{\circ} \mathrm{C}$ with a Peltier system. Temperature jumps from $20^{\circ} \mathrm{C}$ to either $30^{\circ} \mathrm{C}$ or $35^{\circ} \mathrm{C}$ were done at the fastest heating rate $\left(20^{\circ} \mathrm{C} / \mathrm{s}\right)$ and the final temperature was reached within a few seconds. The storage $\left(\mathrm{G}^{\prime}\right)$ and loss $(G$ ') shear moduli were measured at a strain of $1 \%$ that was within the linear response regime. The viscosity of the solutions was measured by shear flow measurements as a function of the shear rate between 1 and $100 \mathrm{~s}^{-1}$. The zero shear viscosity $(\eta)$ was determined from the Newtonian response. Gelation of the samples corresponds to a transition from a liquid to a solid state. This was characterized either in oscillatory shear measurements by $\mathrm{G}^{\prime}$ becoming higher than G" (Figures 2B, 4, 5) or by shear flow measurements by the Newtonian viscosity becoming immeasurably high (Figure 2A). 


\section{Results and Discussion}

\section{Synthesis of dynamic associative BAB copolymers in water}

As summarized in Scheme 2, the synthesis of the BAB triblock copolymers was achieved in one-pot and in the absence of organic solvents. A bifunctional PDMAc macroRAFT agent was synthesized in a first polymerization step performed initially in the bulk and then, after dilution with water, in aqueous solution. In the second polymerization step, the two B-blocks were obtained through the dispersion copolymerization in water of DAAm with various amounts of DMAc using the RAFT-PISA technology.

\section{1st $^{\text {step: PDMAc macroRAFT agent synthesis }}$}

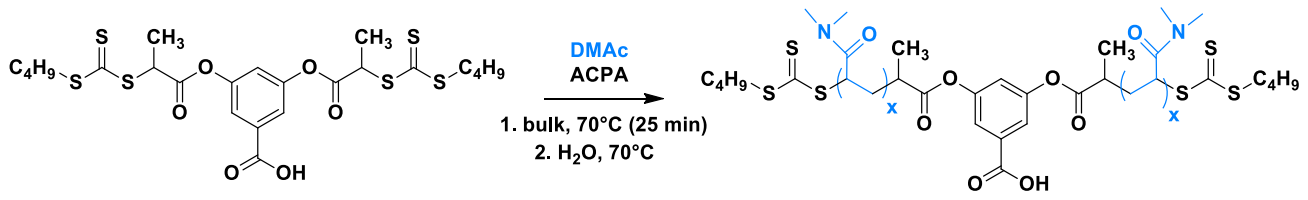

DMAc

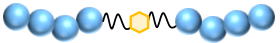

Benzoic acid (= BA)

PDMAc macroRAFT

$2^{\text {nd }}$ step: Aqueous dispersion copolymerization of DMAc and DAAm (PISA)

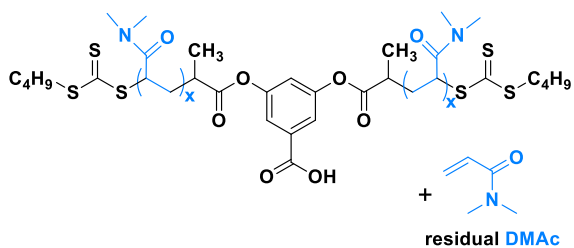

DAAm

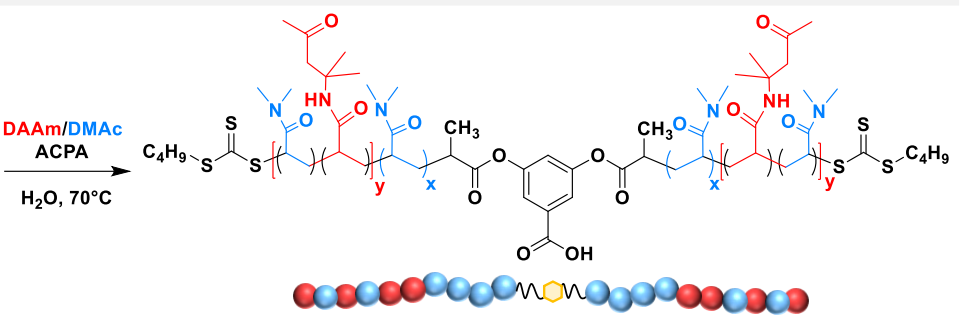

BAB copolymer

Scheme 2. Reaction scheme for the two-step synthesis of $\left[\mathrm{C}_{4}-\mathrm{P}(\mathrm{DAAm}-c o-\mathrm{DMAc})-b\right.$ PDMAc] $]_{2}$-BA completely in water.

PDMAc macroRAFT agents with three different number-average degrees of polymerization, $D P_{\mathrm{n}, \mathrm{A} \text { block }}=80,180$ and 400, were targeted (for experimental conditions and results see Table S1). A symmetrical bifunctional trithiocarbonate RAFT agent, $\left(\mathrm{C}_{4}-\mathrm{TTC}\right)_{2}$-BA, with a 3,5disubstitued benzoic acid (BA) as a central leaving group $\mathrm{R}$, and $\mathrm{S}_{-} \mathrm{C}_{4} \mathrm{H}_{9}$ as the $\mathrm{Z}$ groups at both ends of the molecule $(\mathrm{R}=$ leaving group and $\mathrm{Z}=$ stabilizing group, see Scheme 2 ) was chosen as the chain transfer agent and synthesized according to a previously established protocol. ${ }^{43}$ Since this RAFT agent was not soluble in water, the polymerization of DMAc was started in bulk conditions. As the polymerization proceeds, a few monomer units are added, leading to a water-soluble oligomeric RAFT agent and an increase in viscosity of the polymerization 
medium. In order to decrease the viscosity and ensure a good polymerization control, water was added at about 50\% monomer conversion. ${ }^{1} \mathrm{H}$ NMR analysis indicated that high monomer conversions were reached (>90\%), generally within less than 2 hours. As shown by the SEC overlay of the final PDMAc macroRAFT agents (see Figure S2), monomodal signals with narrow molar mass distribution $(\nexists \sim 1.2)$ were obtained. Moreover, the experimental molar masses determined by ${ }^{1} \mathrm{H}$ NMR were close to the theoretical ones indicating that a good control over the polymerizations was achieved. The PDMAc macroRAFT agent solutions, containing residual amount of DMAc, were then used without further purification in the $2^{\text {nd }}$ polymerization step.

In the $2^{\text {nd }}$ polymerization step, the PDMAc macroRAFT agents were chain-extended in water by statistical copolymerization of a mixture of DAAm and DMAc (see experimental conditions and results summarized in Table 1). To do so, the desired amount of DAAm and DMAc and a new load of ACPA were added to the previously prepared PDMAc macroRAFT agent aqueous solutions. The mol\% of DMAc in the B-segments was tuned from 0 to $50 \%$ by adjusting the added quantity of hydrophilic comonomer DMAc, while keeping constant the total monomer concentration at $12 \mathrm{wt} \%$. We stress that the macroRAFT agent contains a benzoic acid (BA) moiety in its center. In our previous work on $\mathrm{BAB}$ triblock copolymers comprising pure PDAAm B blocks, we had shown that the degree of ionization of the BA moiety strongly affects both the colloidal stability of the dispersion and the control over the polymerization. ${ }^{38,39}$ Phase separation indeed occurred in the absence of charge in the central BA moiety because of intermicellar attraction caused by the ability of the $\mathrm{BAB}$ triblock copolymers to form bridges between micelles. This resulted in loss of colloidal stability (and occasionally loss of control over molar mass). In contrast, when the BA moiety was partially ionized the phase separation was suppressed thanks to the electrostatic repulsion between the micelles. Consistently, phase separation and loss of control were also observed here for $\alpha=47 \%(\mathrm{pH}=4.2)$ with $\mathrm{DP}_{\mathrm{n}}(\mathrm{A}$ block) 100, $\mathrm{DP}_{\mathrm{n}}(\mathrm{B}$ blocks) $~ 190$ and 6.4 mol\% DMAc in the B blocks (see Table S2 entry A and Figure S3), whereas neither colloidal stability nor control were lost at $\alpha=85 \%$ (corresponding to $\mathrm{pH}=5$, see Table S2 entry B and Figure S3). To insure colloidal stability and control over the molar masses in the present study, the ionization degree was therefore kept constant at $\alpha=85 \%$ by adjusting the $\mathrm{pH}$ to a constant value of 5 .

As summarized in Table 1, essentially three PDMAc macroRAFT agents, differing in their block lengths $\left(D P_{\mathrm{n}, \mathrm{A} \text { block }} \sim 80,180\right.$ and 400$)$, were used in three series of aqueous dispersion copolymerizations (series I, II, III). Within each series, the hydrophilic content within the hydrophobic B blocks (mol\% DMAc) was varied from 0 to $50 \%$ and two hydrophobic block 
lengths (overall $D P_{\mathrm{n}, \mathrm{B}}$ blocks $\sim 100$ or 400 ) were targeted. The BAB triblock copolymers are noted "X-Y-Z", where " $\mathrm{X}$ " stands for $D P_{\mathrm{n}}$ of the PDMAc macroRAFT used (block A), "Y" indicates the total $D P_{\mathrm{n}}$ of both B blocks $\left(D P_{\mathrm{n}, \mathrm{B} \text { blocks }}=2 \times D P_{\mathrm{n}, \mathrm{B} \text { arm }}\right)$ and " $\mathrm{Z}$ " indicates the mol\% of DMAc in the hydrophobic block.

To study the incorporation of the monomers within the polymer chain, we first monitored the kinetics of a typical copolymerization of DAAm and DMAc (experiment 80-100-33, Table 1) by ${ }^{1} \mathrm{H}$ NMR (Figure S4). It showed that DAAm and DMAc were consumed at a similar rate, corroborating that the B-blocks were composed of P(DAAm-ran-DMAc) random copolymers. These results are consistent with the literature reporting that the reactivity of DMAc and DAAm is very similar in water. ${ }^{40}$

The individual monomer conversions at the end of the polymerizations are reported in Table 1. For all dispersion polymerizations high conversions for both DAAm and DMAc were reached within 2 hours. The SEC analysis showed that efficient chain extensions and relatively low dispersity values $(<1.3)$ were obtained in all studied conditions, indicating that triblock copolymers were formed in all cases (see Figure S5). 
Table 1. Aqueous dispersion copolymerizations of DAAm and DMAc at $\alpha=85 \%$ in the presence of PDMAc macroRAFT. ${ }^{a}$

\begin{tabular}{|c|c|c|c|c|c|c|c|c|c|}
\hline Entry & $\begin{array}{c}M_{\mathrm{n}, \mathrm{NMR}}{ }^{b} \\
\text { macroRAFT }^{\left(\mathrm{kg} \mathrm{mol}^{-1}\right)}\end{array}$ & $\begin{array}{l}\text { mol\% } \\
\text { DMAc }^{c}\end{array}$ & $\begin{array}{c}\text { time } \\
(\mathbf{m i n})\end{array}$ & $\begin{array}{c}\text { DMAc } \\
\text { conv. } \\
(\%)^{d}\end{array}$ & $\begin{array}{l}\text { DAAm } \\
\text { conv. } \\
(\%)^{d}\end{array}$ & $\begin{array}{c}\text { total } \\
D P_{n, t h}{ }^{e} \\
(\mathrm{~B} \text { blocks })\end{array}$ & $\begin{array}{c}M_{\mathrm{n}, \mathrm{th}}{ }^{e} \\
\left(\mathrm{~kg} \mathrm{~mol}^{-1}\right)\end{array}$ & $\begin{array}{l}M_{\mathrm{n}, \mathrm{PMMA}^{f}} \\
\left(\mathrm{~kg} \mathrm{~mol}^{-1}\right)\end{array}$ & $\boldsymbol{\oplus}^{f}$ \\
\hline \multicolumn{10}{|c|}{ Series $I-D P_{n, A}$ block $\sim 80$} \\
\hline $80-100-0 *$ & 9.2 & 0 & 187 & - & 97 & 86 & 23.8 & 17.5 & 1.23 \\
\hline $80-100-20$ & 8.2 & 20 & 120 & 100 & 100 & 108 & 24.8 & 20.3 & 1.19 \\
\hline $80-100-33$ & 8.2 & 33 & 120 & 99 & 98 & 111 & 24.1 & 20.9 & 1.18 \\
\hline $80-100-50$ & 8.2 & 50 & 124 & 98 & 99 & 111 & 23.1 & 19.2 & 1.21 \\
\hline $80-400-42$ & 8.2 & 42 & 134 & 98 & 100 & 391 & 63.2 & 54.3 & 1.23 \\
\hline \multicolumn{10}{|c|}{ Series $I I-D P_{n, \text { A block }} \sim 180$} \\
\hline 180-100-20 & 18.5 & 20 & 120 & 85 & 88 & 96 & 33.4 & 29.3 & 1.22 \\
\hline $180-100-33$ & 18.4 & 33 & 120 & 88 & 90 & 98 & 32.8 & 25.0 & 1.21 \\
\hline $180-100-50$ & 18.4 & 50 & 120 & 94 & 96 & 105 & 32.5 & 25.0 & 1.20 \\
\hline $180-400-20$ & 18.4 & 20 & 137 & 100 & 100 & 390 & 78.9 & 70.5 & 1.28 \\
\hline $180-400-42$ & 18.5 & 42 & 120 & 98 & 99 & 391 & 73.0 & 62.3 & 1.21 \\
\hline \multicolumn{10}{|c|}{ Series $I I I-D P_{n, \text { A block }} \sim 400$} \\
\hline 400-100-50 & $40.0^{g}$ & 50 & 137 & 100 & 100 & 111 & 54.9 & 46.6 & 1.17 \\
\hline $400-400-42$ & $40.0^{g}$ & 42 & 137 & 100 & 100 & 388 & 94.2 & 74.8 & 1.26 \\
\hline
\end{tabular}

${ }^{a}$ Polymerization conditions: $\mathrm{T}=70{ }^{\circ} \mathrm{C}$, [monomers $]_{0}=12 \mathrm{wt} \%, \alpha(\mathrm{BA})=85 \%$ (corresponding to $\mathrm{pH}=5$ ), $\left[\mathrm{ACPA} \text { added in } 2^{\text {nd }} \text { polymerization step }\right]_{0}=0.76 \mathrm{mmol} \mathrm{L}^{-1}(\mathrm{we}$ note that the majority of ACPA added in the $1^{\text {st }}$ polymerization step was not consumed at the end of step 1, and is therefore also present at the beginning of the $2^{\text {nd }}$ polymerization step); more detailed synthetic conditions are reported in Table S3. ${ }^{b}$ Number-average molar mass $M_{\mathrm{n}, \mathrm{NMR}}$ of PDMAc macroRAFT, determined by ${ }^{1} \mathrm{H}$ NMR. ${ }^{c}$ Molar percentage of DMAc in the two B blocks. mol\% DMAc $=\left[\mathrm{n}\left(\mathrm{DMAc}_{\text {residual }}\right)+\mathrm{n}\left(\mathrm{DMAc}_{\text {added }}\right)\right] /\left[\mathrm{n}(\mathrm{DAAm})+\mathrm{n}\left(\mathrm{DMAc}_{\text {residual }}\right)+\mathrm{n}\left(\mathrm{DMAc}_{\text {added }}\right)\right] \times 100 .{ }^{d}$ Monomer conversion determined by ${ }^{1} \mathrm{H}$ NMR. ${ }^{e}$ Total theoretical number-average degree of polymerization, $D P_{\mathrm{n}, \mathrm{B}}$ blocks, and number-average molar mass, $M_{\mathrm{n}, \mathrm{th}}$, calculated using the experimental conversion and $M_{\mathrm{n}, \mathrm{NMR}}$ for the PDMAc block. ${ }^{f}$ Number-average molar mass $M_{\mathrm{n}, \mathrm{PMMA}}$ and dispersity, $\theta$, determined by SEC in DMF $\left(+\operatorname{LiBr} 1 \mathrm{~g} \mathrm{~L}^{-1}\right)$ with a PMMA calibration. ${ }^{g}$ Theoretical number-average molar mass $M_{\mathrm{n}}$ calculated using the experimental conversion. * For this polymerization the macroRAFT agent was synthesized in DMF, purified by precipitation and added as a solid in the polymerization medium..$^{38} \mathrm{BAB}$ triblock copolymers are noted "X-Y-Z", where "X" stands for the $D P_{\mathrm{n}, \mathrm{A} \text { block }}$ of the PDMAc macroRAFT used, "Y" indicates the overall $D P_{\mathrm{n}, \mathrm{B} \text { blocks }}$ and "Z", the mol\% of DMAc in the hydrophobic blocks. 


\section{Influence of the copolymer composition on viscosity}

For all studied experimental conditions (Table 1), stable dispersions were obtained that were transparent or slightly turbid. By the naked eye, strong differences in viscosity were however observed by simple tilt tests: some samples exhibited a viscosity similar to water, whereas others were highly viscous or formed freestanding gels (see Figure 1). In order to assess qualitatively the impact of the DMAc content in the B blocks on the viscosity, two series of copolymers dispersions were studied, differing in the length of the A block (series I : $D P_{\mathrm{n}, \mathrm{A}}$ block $\sim 80$; series II: $D P_{\mathrm{n}, \text { A block }} \sim 180$ ), while keeping constant the overall $D P_{\mathrm{n}}$ of the B blocks $\left(D P_{\mathrm{n}, \mathrm{B}}\right.$ blocks $\left.\sim 100\right)$. Within each series, the DMAc content in the B blocks was systematically varied. For comparison, the final dispersions were diluted to the same polymer concentration of $18 \mathrm{wt} \%$ and macroscopically observed at $25{ }^{\circ} \mathrm{C}$. Selected samples were also analyzed by rheology.

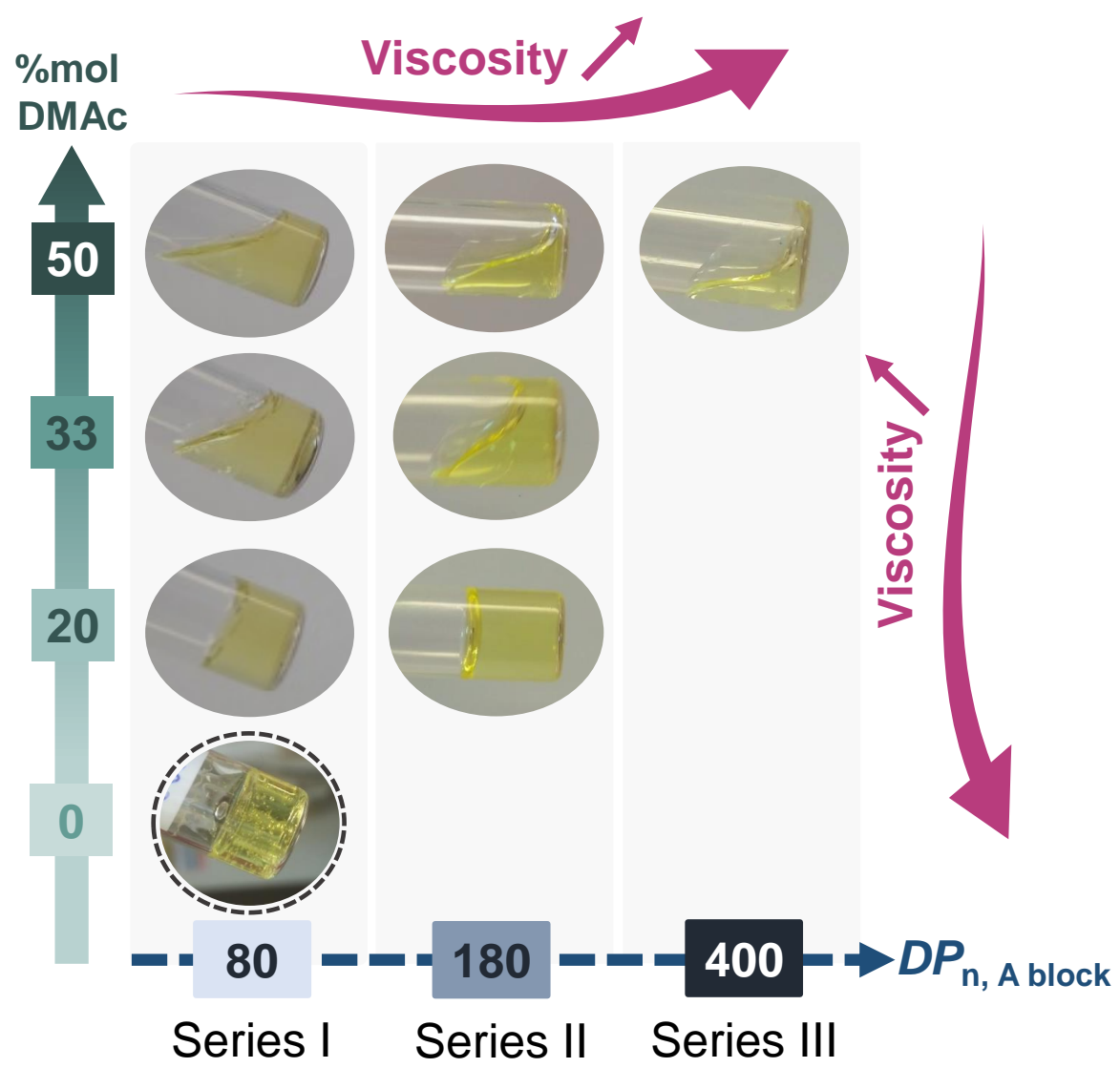

Figure 1. Macroscopic aspect of triblock copolymers with total $D P_{\mathrm{n}, \mathrm{B}}$ blocks $\sim 100$ at $18 \mathrm{wt} \%$ in water, at $25{ }^{\circ} \mathrm{C}$ observed $5 \mathrm{~s}$ after vial tilting. The dotted circle indicates a gel-like dispersion.

As illustrated in Figure 1, for both series, visually the viscosity significantly decreased with increasing DMAc content in blocks B. Within series I (polymers with the relatively short A 
block), copolymers with a DMAc content $\geq 33 \mathrm{~mol} \%$ were liquid, whereas the sample with the lowest DMAc content (20 mol\%, 80-100-20) was highly viscous. In contrast, the reference sample without DMAc in the B blocks, 80-100-0, formed a transparent brittle gel, attributed to the formation of a frozen system. ${ }^{39}$ Shear tests performed at $5 \mathrm{wt} \%$ and $30{ }^{\circ} \mathrm{C}$ on samples 180 100-20 and 180-100-33, confirmed the strong impact of the DMAc content on sample viscosity (see results in Table S4). TEM and cryo-TEM analyses (performed at 0.1 and 1 wt \%, respectively) showed that the dispersions - regardless of the DMAc content - were generally constituted of sub-50 nm spherical aggre gates (see Figure S6 and Table S5), in agreement with previous results. ${ }^{38,46} \mathrm{We}$ can therefore conclude that the changes in viscosity were not related to a change in particle morphology. The observations - together with previous results on samples containing pure PDAAm B blocks ${ }^{39}$ - rather suggest that a percolated network of bridged flower-like micelles is formed, and that increasing the DMAc content in the B blocks decreases the lifetime of the B blocks within the micellar cores and/or decreases the number of elastically active chains, thereby decreasing the viscosity of the samples.

Furthermore, the influence of the overall length of the hydrophilic and the hydrophobic blocks on the sample viscosity was also visually assessed. At $18 \mathrm{wt} \%$, a significant increase in viscosity was observed when the $D P_{\mathrm{n}}$ of the hydrophilic A block $\left(D P_{\mathrm{n}, \mathrm{A}}\right.$ block$)$ was increased (from 80 to 180 to 400 , keeping constant the DMAc content and the overall $D P_{\mathrm{n}}$ of the B blocks, set to either $D P_{\mathrm{n}, \mathrm{B} \text { blocks }} \sim 100$ or 400, see Figure 1 and Figure S7). $\$$ These visual observations were further confirmed by rheological analyses performed at $5 \mathrm{wt} \%$ on samples 80-100-33 and 180100-33 (see results in Table S4). These results are consistent with the fact that longer hydrophilic blocks should favor percolation of the network at low concentration, thereby increasing the number of elastically active chains and therefore the viscosity.

Increasing the length of the B block at constant DMAc content also increased viscosity. For instance, copolymer 180-100-20, with a $D P_{\mathrm{n}, \mathrm{B}}$ block $\sim 100$, led to a highly viscous dispersion, whereas a brittle gel was formed for copolymer 180-400-20, with $D P_{\mathrm{n}, \mathrm{B}}$ blocks $\sim 400$ (see Figure 1 and Figure S7). $\ddagger$ This should be attributed to a decrease of the exchange rate of the B blocks (with increasing length of the outer blocks), ${ }^{21,47}$ although an increase of the number of elastically active chains with longer B blocks cannot not be excluded.

\footnotetext{
$\$$ The samples were compared at the same weight percentage of $18 \%$, i.e. at slightly different molar concentration. In order to compare sample viscosity, the molar concentration is determining. Considering that the molar concentration is actually lower for the samples with a longer hydrophilic A or longer hydrophobic B blocks, we can conclude that there is truly an increase of viscosity with increasing length of either type of block.
} 


\section{Thermoresponsive behavior}

\section{a) Non-monotonic viscosity change with temperature}

Vial inversion tests on concentrated samples (at $\mathrm{C}=18 \mathrm{wt} \%$ ) performed at different temperatures, $\mathrm{T}=5,25$ or $70{ }^{\circ} \mathrm{C}$, revealed that all samples exhibited visually a temperaturedependent viscosity. Generally, the viscosity increased with temperature independently of the DMAc content and block lengths (see Figure S8 for some examples). The only exception was the sample without DMAc in the B blocks (entry 80-100-0 in Table 1), which remained a brittle gel at all studied temperatures. The thermo-responsiveness of the copolymers was also visually observed at lower polymer concentration. At a polymer concentration of $5 \mathrm{wt} \%$, sample 180100-20, exhibited an unexpected non monotonic thermoresponsive behavior: the viscosity at $\mathrm{T}$ $=25{ }^{\circ} \mathrm{C}$ was higher than that at $5{ }^{\circ} \mathrm{C}$ and $70{ }^{\circ} \mathrm{C}$ (see video in the Supporting Information). Viscosity measurements at the same concentration confirmed these visual observations and revealed a maximal viscosity at about $22^{\circ} \mathrm{C}$ (Figure S9).

For most of the other samples, it was impossible to assert significant viscosity changes with temperature by the naked eye at $\mathrm{C}=5 \mathrm{wt} \%$. The thermo-responsiveness of sample 180-100-33, containing a slightly higher DMAc content than 180-100-20, was therefore further investigated by rheology. Figure 2A shows the low shear viscosity as a function of temperature for different polymer concentrations (C). At $\mathrm{C} \leq 3 \mathrm{wt} \%$, the viscosity was close to that of water (at least for $\mathrm{T} \geq 20{ }^{\circ} \mathrm{C}$, data not shown), whereas at $\mathrm{C} \geq 5 \mathrm{wt} \%$ the viscosity was significantly higher. At $\mathrm{C}$ $=5$ and $10 \mathrm{wt} \%$ the viscosity increased first with increasing temperature reaching a maximum before decreasing again. At 14 and $24 \mathrm{wt} \%$, the systems gelled above a critical temperature (Figure 2A), which will be discussed in more detail in Section 3b. Compared to sample 180100-20, containing a slightly lower amount of DMAc in the B block, at $5 \mathrm{wt} \%$, the temperature for which the viscosity is maximal was shifted from $22{ }^{\circ} \mathrm{C}$ (Figure S9) towards higher temperature $\left(40{ }^{\circ} \mathrm{C}\right)$. This unexpected non-monotonic behavior is thus a general property of these $\mathrm{BAB}$ copolymers, and the transition temperature is dependent of the DMAc content in the B blocks. Furthermore, the temperature and the viscosity at the maximum increased with increasing concentration. 
A

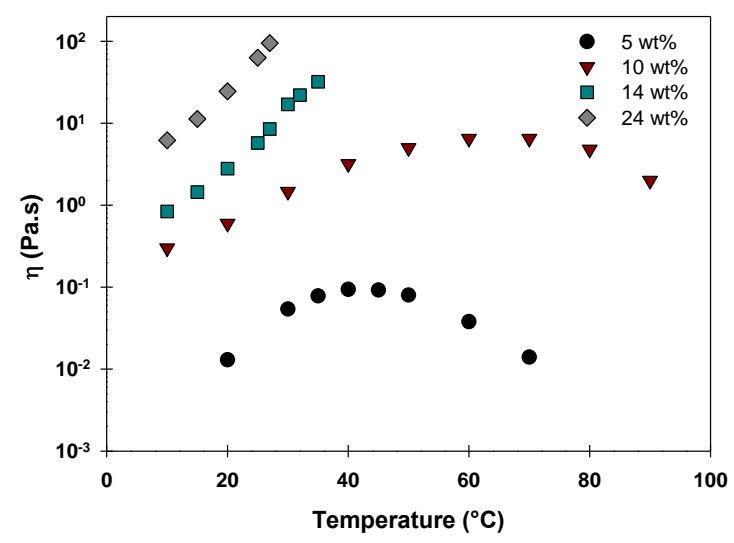

B

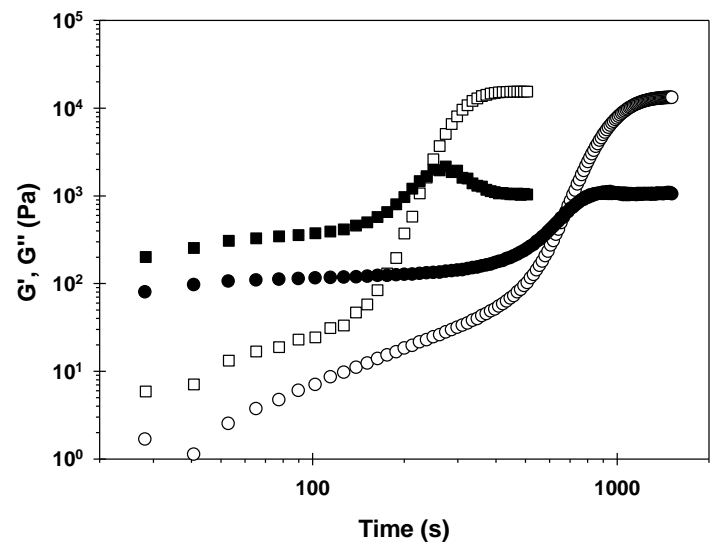

Figure 2. (A) Dependence of the viscosity $(\eta)$ in the Newtonian regime as a function of the temperature for different polymer concentrations for sample 180-100-33 (see Table 1): $5 \mathrm{wt} \%$ $(\bullet), 10 \mathrm{wt} \%(\boldsymbol{\nabla}), 14 \mathrm{wt} \%(\boldsymbol{\square})$ and $24 \mathrm{wt} \%(\diamond)$. At $14 \mathrm{wt} \%$ and $24 \mathrm{wt} \%$, the viscosity became immeasurable large above $\mathrm{T}=35^{\circ} \mathrm{C}$ or $28^{\circ} \mathrm{C}$, respectively. This marked the critical gelation temperature discussed in section $2 \mathrm{~b}$. (B) Evolution of the storage and loss moduli as a function of time obtained after heating $24 \mathrm{wt} \%$ dispersions from $20{ }^{\circ} \mathrm{C}$ towards $30^{\circ} \mathrm{C}\left(\mathrm{G}^{\prime} \bigcirc\right.$ and $\left.\mathrm{G}^{\prime} \cdot \mathbf{O}\right)$ and $35^{\circ} \mathrm{C}\left(\mathrm{G}^{\prime} \square\right.$ and $\left.\mathrm{G}^{\prime} \boldsymbol{\mathrm { D }}\right)$ with an applied strain of $1 \%$ and frequency of $1 \mathrm{~Hz}$. Gelation is observed when G' steeply increases and becomes higher than G".

Cryo-TEM analyses of the sample at 25,40 and $70{ }^{\circ} \mathrm{C}$ (see Figure S11) did not show any morphological transition upon heating. Spherical aggregates were obtained for all temperatures, and no significant change in diameter could be assessed (Table S5). Thus, we can suppose that the non-monotonic variation of viscosity with $\mathrm{T}$ at low polymer concentration was caused by the formation of a percolated network of bridged flower-like spherical micelles for which the bond lifetime and/or the number of elastically active bridges has a maximum with T. It is clear that for the systems studied here at $\mathrm{C} \leq 10 \mathrm{wt} \%, \mathrm{G}$ ” $\propto \mathrm{f}$, even at the highest accessible frequency $\mathrm{f}$, which means that the bond lifetime was shorter than $0.02 \mathrm{~s}(\mathrm{t}=1 /(2 \pi \mathrm{f})$ with $\mathrm{f}=10 \mathrm{~Hz}) \cdot{ }^{1} \mathrm{H}$ NMR studies of sample 180-100-33 conducted at different temperatures in $\mathrm{D}_{2} \mathrm{O}$ at $2.5 \mathrm{wt} \%$ (see Figure 3 and Figure S12 for more detailed information), also showed a non-monotonic evolution of the intensity of the signals characterizing the PDAAm block. When the sample was heated from $5{ }^{\circ} \mathrm{C}$ to $35^{\circ} \mathrm{C}$ a decrease of the intensity of the PDAAm signals (signals $i$ and $\boldsymbol{d}$ in Figure S12) was observed, while further heating to $80^{\circ} \mathrm{C}$ led to an increase in intensity. The minimum in intensity around $35^{\circ} \mathrm{C}$ corresponds to a minimum of chain mobility of the $\mathrm{B}$ blocks. The strong correlation between the minimum of mobility observed by ${ }^{1} \mathrm{H}$ NMR for the 
B blocks and the maximum of macroscopic viscosity of the sample (Figure 3) suggests that the latter phenomenon is certainly related to the effect of temperature on the B blocks, but we cannot exclude that the A block is also affected by the temperature.

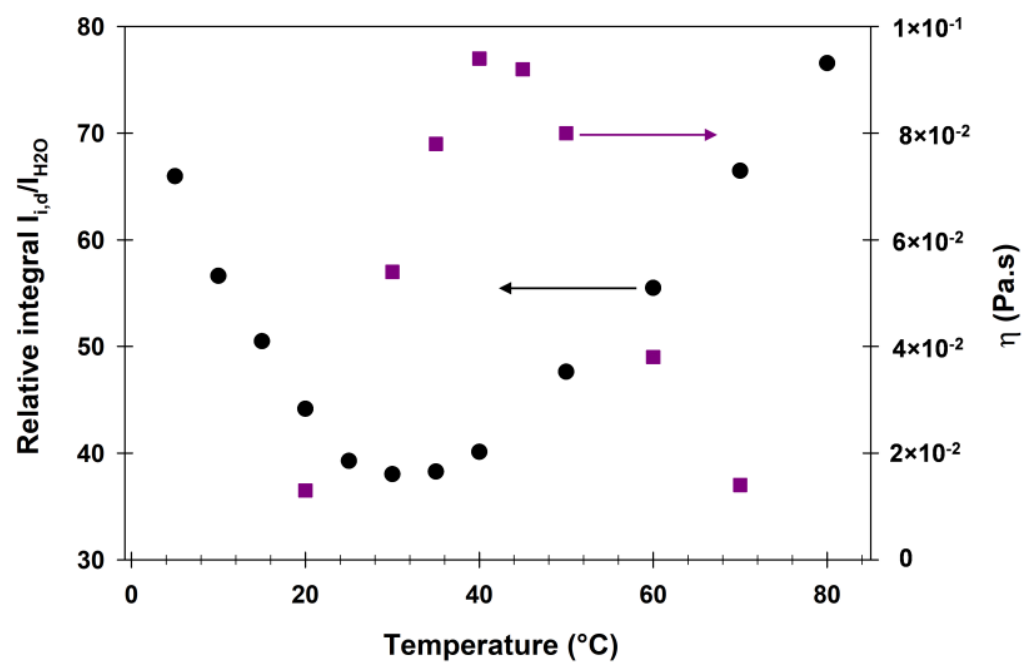

Figure 3. Temperature-responsiveness of sample 180-100-33: Temperature-dependent evolution of the relative integration of protons " $i$ and $e$ " characteristic of PDAAm (see Figure S12) observed by NMR spectroscopy at $2.5 \mathrm{wt} \%$ in $\mathrm{D}_{2} \mathrm{O}(0)$ and viscosity at $5 \mathrm{wt} \%$ in water (ם).

\section{b) Temperature-induced gelation at higher concentrations}

At $\mathrm{C}=14$ and $24 \mathrm{wt} \%$ the viscosity increased until the system gelled above a critical temperature of 35 and $28{ }^{\circ} \mathrm{C}$, respectively (Figure $2 \mathrm{~A}$ ). At $\mathrm{C}=24 \mathrm{wt} \%$, the gelation was investigated at 30 and $35^{\circ} \mathrm{C}$ (i.e. slightly above the gelation temperature) starting from a liquid state at $20^{\circ} \mathrm{C}$. As displayed in Figure $2 \mathbf{B}$ the gelation was characterized by an increase of G' with time exceeding $G$ " before reaching a steady state value $\mathrm{G}_{\mathrm{el}} \approx 1.5 \times 10^{4} \mathrm{~Pa}$. The rate of gelation depended on the temperature at which it occurred as shown by temperature-jumps from a liquid state at $20^{\circ} \mathrm{C}$ to a final temperature of either 30 or $35^{\circ} \mathrm{C}$. The rate of gelation indeed decreased significantly when the final temperature was decreased from $35^{\circ} \mathrm{C}$ to $30^{\circ} \mathrm{C}$, which is indeed very close to the critical gelation temperature $\left(28{ }^{\circ} \mathrm{C}\right.$ at $\left.24 \mathrm{wt} \%\right)$. As previously mentioned, the system did not gel upon heating from a lower temperature up to $25^{\circ} \mathrm{C}$, while the gel formed at higher temperature melted, i.e. became a viscous solution, when the temperature was decreased to $25^{\circ} \mathrm{C}$. Figure 4 shows the frequency dependence of the shear 
moduli at steady state at different temperatures. For the gels $\left(\mathrm{T} \geq 28^{\circ} \mathrm{C}\right) \mathrm{G}$ ' was larger than $\mathrm{G}$ ' and almost independent of the frequency over the whole range that was explored and G" showed a weak minimum. For the viscous liquids formed at lower temperatures $\left(T=25^{\circ} \mathrm{C}\right) \mathrm{G}$ " was larger than $G^{\prime}$ and increased linearly with increasing frequency.

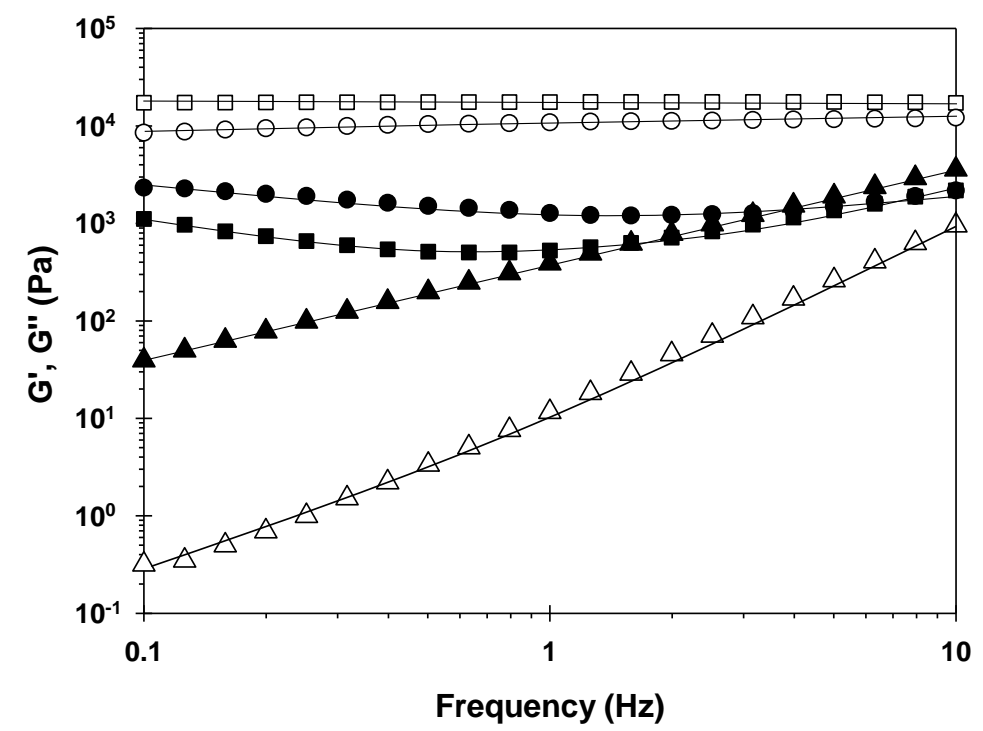

Figure 4. Frequency dependence of the storage and loss moduli for a $24 \mathrm{wt} \%$ dispersion of copolymer 180-100-33 at an applied strain of $1 \%$ and at different temperatures: $25^{\circ} \mathrm{C}$ (G' $\triangle$ and $\left.\mathrm{G}^{\prime} \boldsymbol{\Delta}\right), 28^{\circ} \mathrm{C}\left(\mathrm{G}^{\prime} \bigcirc\right.$ and $\left.\mathrm{G}^{\prime}, \mathbf{O}\right)$ and $35^{\circ} \mathrm{C}\left(\mathrm{G}^{\prime} \square\right.$ and $\left.\mathrm{G}^{\prime \prime} \mathbf{\square}\right)$. The solid lines are guides to the eye.

When gels that were melted by cooling to $20^{\circ} \mathrm{C}$ were heated again to $30^{\circ} \mathrm{C}$, they formed gels again in exactly the same manner as when they were heated the first time (data not shown). This shows that the system was fully temperature reversible. Interestingly, when the gels were broken up at a fixed temperature by applying a strong shear flow $\left(1000 \mathrm{~s}^{-1}\right)$, they fully recovered after stopping the shear applied (Figure 5). The recovery after shear was found to be faster than the temperature-induced gel formation (heating from $20{ }^{\circ} \mathrm{C}$ to $30{ }^{\circ} \mathrm{C}$ ), which suggests that strong shear does not fully rejuvenate the system as cooling to $20^{\circ} \mathrm{C}$ would do. 


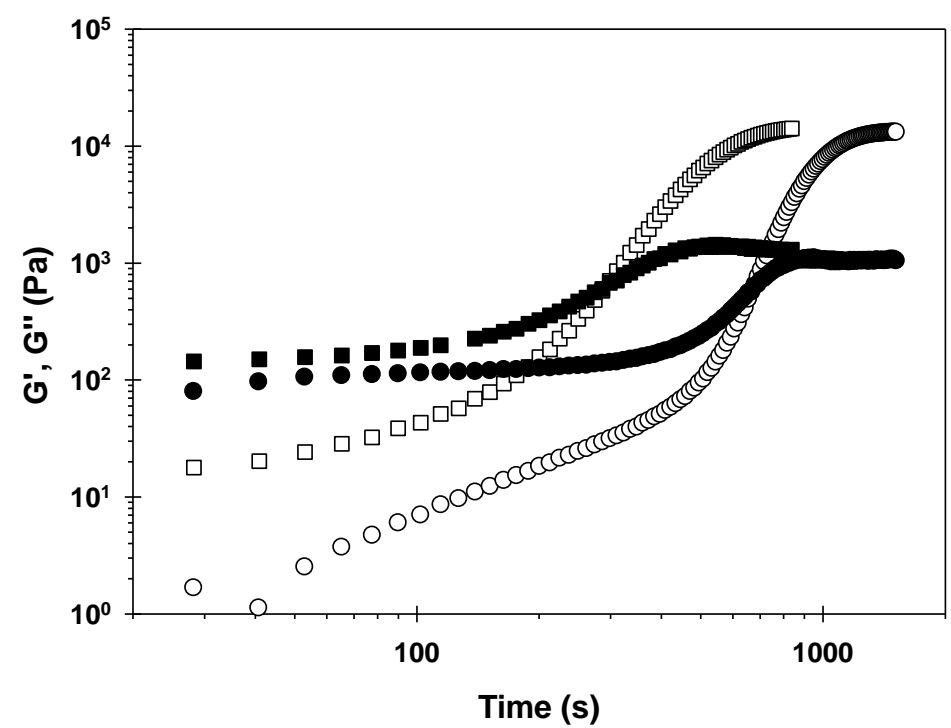

Figure 5. Gel formation over time at $30{ }^{\circ} \mathrm{C}$ of a 24 wt\% dispersion of copolymer 180-10033 (see Table 1): Gel formation by heating the liquid dispersion $\left(\mathrm{G}^{\prime} \bigcirc\right.$ and $\left.\mathrm{G}^{\prime} \mathrm{O}\right)$ from 20 ${ }^{\circ} \mathrm{C}$ to $30^{\circ} \mathrm{C}$. Gel recovery after being broken under high shear $\left(1000 \mathrm{~s}^{-1}\right)$ at $30{ }^{\circ} \mathrm{C}\left(\mathrm{G}^{\prime} \square\right.$ and G' $\square$ ). The time evolution was measured at $30{ }^{\circ} \mathrm{C}$ with an applied strain of $1 \%$ (linear regime) and a frequency of $1 \mathrm{~Hz}$.

As mentioned above, a gel is also formed at $\mathrm{C}=14 \mathrm{wt} \%$ for $\mathrm{T} \geq 35^{\circ} \mathrm{C}$ (Figure $2 \mathrm{~A}$ ), but the gel was much weaker than at $\mathrm{C}=24 \mathrm{wt} \%$ (see Figure S10). The gel formed at $\mathrm{C}=14 \mathrm{wt} \%$ melted at $70{ }^{\circ} \mathrm{C}$. G' was found to increase slightly with increasing temperature from $2 \times 10^{3} \mathrm{~Pa}$ at $40{ }^{\circ} \mathrm{C}$ to $3 \times 10^{3} \mathrm{~Pa}$ at $50{ }^{\circ} \mathrm{C}$, then decreased again to about $2 \times 10^{3} \mathrm{~Pa}$ at $60{ }^{\circ} \mathrm{C}$ and melted at $70{ }^{\circ} \mathrm{C}$. Therefore, the non-monotonous effect of temperature was not only observed on the viscosity at the lowest concentrations, but also on the modulus at the highest concentrations.

A gelation above a critical temperature can in principle occur for a percolated network of BAB triblock copolymers if the raise of temperature increases drastically the strength of hydrophobic interactions in the B blocks so that the bond life time becomes infinitely long (compared to the observation time scale). ${ }^{15,16,25}$ However, if this was the reason for the temperature-induced gelation observed at high concentration $(\mathrm{C} \geq 14 \mathrm{wt} \%)$, this phenomenon would also have been observed at lower concentrations (5 and $10 \mathrm{wt} \%$ ). This was not the case (see section 3.a.), although the viscosity of the polymer solution at $20{ }^{\circ} \mathrm{C}$ (Figure 2A) indeed suggests that a percolated network was already formed at $5 \mathrm{wt} \%$. 
Therefore, we suggest that the gel formation (for $\mathrm{C} \geq 14 \mathrm{wt} \%$ ) is caused by jamming of the micelles as already reported in the literature for other types of associative networks. ${ }^{48,49}$ Such a process is caused by close packing of micelles and occurs only at very high concentrations as observed here (independently of the formation of a transient network). For poly(ethylene oxide) (PEO) end-capped at one or two ends with short alkyl chains, jamming was observed below a critical temperature, ${ }^{48}$ which is the reverse behavior to the system studied here. This was attributed to a decreasing repulsion between the PEO coronas with increasing temperature. Interestingly, an increase of G' with time was observed when the temperature was cooled below the critical temperature, which is indeed similar to what we observed in this study when the temperature was increased above the critical temperature. In addition, the gelation rate was observed to decrease when the temperature was set closer to the critical value similar to what was observed for the present system (Figure 2B).

Considering that the elasticity of gels formed by jamming has a purely entropic origin for flexible polymers such as these, the stiffness of the gels should be related to the number concentration of deformed chains $(v)$ : $\mathrm{G}_{\mathrm{el}}=v \mathrm{kT}$, where $\mathrm{k}$ is Boltzman's constant and $\mathrm{T}$ is the absolute temperature. If all chains are elastically active (deformed) $G_{e l}=\left(R T / M_{n}\right) \times C$, which gives $1.8 \times 10^{4} \mathrm{~Pa}$ at $\mathrm{C}=24 \mathrm{wt} \%$ at $35^{\circ} \mathrm{C}$ close to the experimentally observed value (Figure 4). However, at $\mathrm{C}=14 \mathrm{wt} \%$, the theoretical value at $50{ }^{\circ} \mathrm{C}\left(1.0 \times 10^{4} \mathrm{~Pa}\right)$ is much bigger than the observed value of $\mathrm{G}_{\mathrm{el}}\left(3 \times 10^{3} \mathrm{~Pa}\right)$ suggesting that all micelles were not yet strongly jammed at this concentration (Figure S10). In addition, at C = $14 \mathrm{wt} \%$ the system did not gel at $70{ }^{\circ} \mathrm{C}$, which suggests that the jamming also varies non monotonously with temperature. The origin of the maximum in viscosity and elastic modulus is not fully understood. It could be related to the effect of temperature on the interaction between the hydrophobic groups within the cores and/or to changes in the water content of the cores. More work needs to be done to elucidate the origin of this phenomenon.

\section{Conclusions}

In this work, we developed a synthetic strategy to produce in one-pot by PISA associative BAB triblock copolymers in water without using organic solvents. According to the PISA strategy, assembly occurs simultaneously to chain growth and networks of flower-like micelles are formed. The BAB copolymers are composed of a PDMAc hydrophilic A block and statistical copolymer P(DAAm-co-DMAc) B blocks. Well-defined copolymers containing various DMAc 
contents in the B blocks and different block lengths were synthesized with good polymerization control. Comparison with simple PDAAm- $b$-PDMAc- $b$-PDAAm triblock copolymers clearly showed that the introduction of the DMAc units in the outer blocks made the networks dynamic. We demonstrated that the viscosity of the dispersion was strongly decreased by increasing the amount of DMAc incorporated in the B blocks. This was most probably caused by the formation of a percolated network of bridged flower-like micelles for which the exchange time of the B blocks and/or the number of elastically active chains significantly decreased for high DMAc contents. We also showed that the viscosity could be increased by increasing the length of both the hydrophilic and hydrophobic segments. Most importantly, all the copolymers with DMAc in the B blocks exhibited a reversible thermoresponsive behavior with a maximum of viscosity as a function of temperature. The ${ }^{1} \mathrm{H}$ NMR analyses suggest that this particular behavior is related to the impact of temperature on the B blocks, whose mobility followed a similar nonmonotonous evolution with temperature compared to the macroscopic viscosity. Finally, at high concentrations ( $\mathrm{C} \geq 14 \mathrm{wt} \%)$, a temperature-induced sol-gel transition was observed, which was attributed to jamming of the flower-like micelles and also exhibited a non-monotonous behavior.

Acknowledgments: The authors thank Jean-Michel Guigner (IMPMC, Sorbonne Université) for the cryo-TEM analyses, Patricia Beaunier (LRS, Sorbonne Université) for the TEM analyses and Véronique Peyre (Sorbonne Université, PHENIX) for her advices to determine the $\mathrm{pK}_{\mathrm{a}}$ of the macroRAFT agent. The authors thank the Agence Nationale de la Recherche for funding (PISAForFilms project, ANR-17-CE09-0031-01 \& Dynamic-PISA, ANR-19-CE06-0002-01)

Keywords: PISA, aqueous RAFT dispersion polymerization, BAB triblock copolymers, flower-like micelles, transient network, thermoresponsive copolymers.

\section{References}

1 C. Tsitsilianis, Soft Matter, 2010, 6, 2372-2388.

2 J. Madsen and S. P. Armes, Soft Matter, 2012, 8, 592-605.

3 L. Bromberg, Adv. Drug Deliv. Rev., 1998, 31, 197-221.

4 A. Kikuchi and T. Okano, Adv. Drug Deliv. Rev., 2002, 54, 53-77.

5 F. Ilmain, T. Tanaka and E. Kokufuta, Nature, 1991, 349-350, 400.

6 D. J. Pochan, J. P. Schneider, J. Kretsinger, B. Ozbas, K. Rajagopal and L. Haines, J. Am. Chem. Soc., 2003, 125, 11802-11803. 
7 K. S. Oh, S. K. Han, Y. W. Choi, J. H. Lee, J. Y. Lee and S. H. Yuk, Biomaterials, 2004, 25, 2393-2398.

8 A. Gutowska, B. Jeong and M. Jasionowski, Anat. Rec., 2001, 263, 342-349.

9 S. E. Kirkland, R. M. Hensarling, S. D. McConaughy, Y. Guo, W. L. Jarrett and C. L.

McCormick, Biomacromolecules, 2008, 9, 481-486.

10I. K. Kwon and T. Matsuda, Biomaterials, 2006, 27, 986-995.

11 M. A. Ward and T. K. Georgiou, Polymers, 2011, 3, 1215-1242.

12D. Roy, W. L. A. Brooks and B. S. Sumerlin, Chem. Soc. Rev., 2013, 42, 7214.

13C. Zhao, Z. Ma and X. X. Zhu, Prog. Polym. Sci., 2019, 90, 269-291.

14H. G. Schild, Prog. Polym. Sci., 1992, 17, 163-249.

15T. Annable, R. Buscall, R. Ettelaie and D. Whittlestone, J. Rheol., 1993, 37, 695-726.

16C. Chassenieux, T. Nicolai and L. Benyahia, Curr. Opin. Colloid Interface Sci., 2011, 16, $18-26$.

17M. S. Green and A. V. Tobolsky, J. Chem. Phys., 1946, 14, 80-92.

18F. Tanaka and S. F. Edwards, Macromolecules, 1992, 25, 1516-1523.

19A. Tripathi, K. C. Tam and G. H. McKinley, Macromolecules, 2006, 39, 1981-1999.

20T. Indei, J. Chem. Phys., 2007, 127, 144905.

21 T. Nicolai, O. Colombani and C. Chassenieux, Soft Matter, 2010, 6, 3111-3118.

22C. Charbonneau, C. Chassenieux, O. Colombani and T. Nicolai, Macromolecules, 2011, 44, 4487-4495.

23A. Shedge, O. Colombani, T. Nicolai and C. Chassenieux, Macromolecules, 2014, 47, 2439-2444.

24O. Borisova, L. Billon, M. Zaremski, B. Grassl, Z. Bakaeva, A. Lapp, P. Stepanek and O. Borisov, Soft Matter, 2011, 7, 10824.

25L. Lauber, J. Santarelli, O. Boyron, C. Chassenieux, O. Colombani and T. Nicolai, Macromolecules, 2017, 50, 416-423.

26Y. Mai and A. Eisenberg, Chem. Soc. Rev., 2012, 41, 5969.

27 J. Chiefari, Y. K. (Bill) Chong, F. Ercole, J. Krstina, J. Jeffery, T. P. T. Le, R. T. A.

Mayadunne, G. F. Meijs, C. L. Moad, G. Moad, E. Rizzardo and S. H. Thang,

Macromolecules, 1998, 31, 5559-5562.

28M. J. Derry, L. A. Fielding and S. P. Armes, Prog. Polym. Sci., 2016, 52, 1-18.

29J.-T. Sun, C.-Y. Hong and C.-Y. Pan, Polym. Chem., 2013, 4, 873-881.

30J. Rieger, Macromol. Rapid Commun., 2015, 36, 1458-1471.

31 Y. Pei, A. B. Lowe and P. J. Roth, Macromol. Rapid Commun., 2017, 38, 1600528.

32B. Charleux, G. Delaittre, J. Rieger and F. D’Agosto, Macromolecules, 2012, 45, 67536765.

33 S. L. Canning, G. N. Smith and S. P. Armes, Macromolecules, 2016, 49, 1985-2001.

34M. Lansalot, J. Rieger and F. D'Agosto, in Macromolecular Self-assembly, John

Wiley\&Sons, Inc., 2016, pp. 33-82.

35 W. Wang, C. Gao, Y. Qu, Z. Song and W. Zhang, Macromolecules, 2016, 49, 2772-2781.

36C. Gao, S. Li, Q. Li, P. Shi, S. A. Shah and W. Zhang, Polym. Chem., 2014, 5, 6957-6966.

37Y. Qu, S. Wang, H. Khan, C. Gao, H. Zhou and W. Zhang, Polym. Chem., 2016, 7, 19531962.

38P. Biais, P. Beaunier, F. Stoffelbach and J. Rieger, Polym. Chem., 2018, 9, 4483-4491.

39P. Biais, O. Colombani, L. Bouteiller, F. Stoffelbach and J. Rieger, Polym. Chem., 2020, 11, 4568-4578.

40C. A. Figg, R. N. Carmean, K. C. Bentz, S. Mukherjee, D. A. Savin and B. S. Sumerlin, Macromolecules, 2017, 50, 935-943.

41 P. Shi, H. Zhou, C. Gao, S. Wang, P. Sun and W. Zhang, Polym. Chem., 2015, 6, 49114920. 
42J. Zhou, W. Zhang, C. Hong and C. Pan, Polym. Chem., 2016, 7, 3259-3267.

43 N. Malic and R. A. Evans, Aust. J. Chem., 2006, 59, 763.

44J. Lesage de La Haye, J.-M. Guigner, E. Marceau, L. Ruhlmann, B. Hasenknopf, E. Lacôte and J. Rieger, Chem. - Eur. J., 2015, 21, 2948-2953.

45 J. Dubochet, M. Adrian, J.-J. Chang, J.-C. Homo, J. Lepault, A. W. McDowall and P. Schultz, Q. Rev. Biophys., 1988, 21, 129-228.

46F. D’Agosto, J. Rieger and M. Lansalot, Angew. Chem. Int. Ed., 2020, 10.1002/anie.201911758.

47 A. Halperin and S. Alexander, Macromolecules, 1989, 22, 2403-2412.

48F. Renou, T. Nicolai, L. Benyahia and E. Nicol, J. Phys. Chem. B, 2009, 113, 3000-3007.

49S. Zhang, K. H. Lee, J. Sun, C. D. Frisbie and T. P. Lodge, Macromolecules, 2011, 44, 8981-8989. 